\title{
Video Assisted Thoracoscopic Surgery Ipisilateral Tran-Mediastianal Approach (Using Alzandi Technique) to Contralateral Lung in Patients with Bilateral Lung Lesions
}

\author{
Hassan Alghamdi \\ Department of Surgery_Division of Thoracic \& Esophageal Surgery, King Khalid Hospital, Najran, Saudi Arabia. \\ Email: dr_hassan1117@yahoo.com

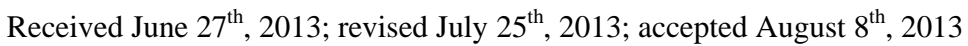 \\ Copyright (C) 2013 Hassan Alghamdi. This is an open access article distributed under the Creative Commons Attribution License, \\ which permits unrestricted use, distribution, and reproduction in any medium, provided the original work is properly cited.
}

\begin{abstract}
Synchronous lung cancer is considered one of the most difficult resection presentations. Bilateral staged thoractomy still exists as the gold standard, as few cases are reported by using median strenotmy. Video-assisted thoracic surgery (VATS) has gained an important role in routine thoracic surgical practice, and staged VATS resection of bilateral lung cancer has been reported with satisfactory results. From here, we describe a patient with bilateral lung cancer in which we successfully resected the contra lateral lung nodule through the ipsilateral VATS approach. To our knowledge, this is the first report of bilateral lung cancer managed with the ipsilateral trans-mediastinal VATS technique (alzandi technique).
\end{abstract}

Keywords: Bilateral; Lung Cancer; Trans-Mediastinal; Ipisilateral; VATS

\section{Introduction}

Single-stage bilateral surgical treatment of synchronous bilateral multiple lung cancer is ideal for cancer curative resection; however, such treatment is considered highly invasive. Bilateral VATS wedge resection has been described for the treatment of bilateral lung nodules. The minimal invasiveness of this technique makes it a good option for those types of patients. There are sporadic studies reporting synchronous multiple adenocarcinomas developing more frequently in the periphery of the lungs, which will lead to easier resections. At this point, we consider another less invasive and cost effective approach. We have shown success in resecting a synchronous lesion in the left upper lobe through the same incisions of VATS, in the right upper lobectomy. This was done in the same sitting with an excellent outcome, by developing the new technique which we call the alzandi technique.

\section{Case}

A 61-year-old woman who had a chest X-ray showed a $2.0 \mathrm{~cm}$ mass on the right lung. A follow up CT scan was done, which showed a right upper lobe speculated mass $(2.8 \mathrm{~cm})$ (Image A), and a $16 \mathrm{~mm}$ mass on the medial left upper lobe (Image B). The mass on the left side was juxtaposed to the mediastinal pleural reflection, anterior to the great vessels. A pulmonary function test indicated decreased pulmonary function (FEV1 62\% (2.581) and DLCO 47\%). A fine needle biopsy of the right nodule was suggestive of malignancy. Moreover, due to her impaired pulmonary function, and the unique location of the left mass, a right upper lobectomy and limited resection on the left using trans-mediastinal VATS resection (alzandi technique ) was chosen to preserve lung function, while offering the advantages of reduced invasiveness and pain, shorter surgical duration, and favorable cosmetic results.

The surgery was discussed and consent was received. The patient was placed under general anesthesia via double-lumen endotracheal intubation. Then, a flexible bronchoscopy was performed which showed no significant endobronchial lesions. A standard right VATS upper lobectomy was performed, and then resection of the left upper lobe was carried out using the trans-mediastinal approach (alzandi technique). The patient was admitted for 3 days post-surgery. She was active and mobile when discharged. The follow up CT scan after 6 month showed no recurrence. 

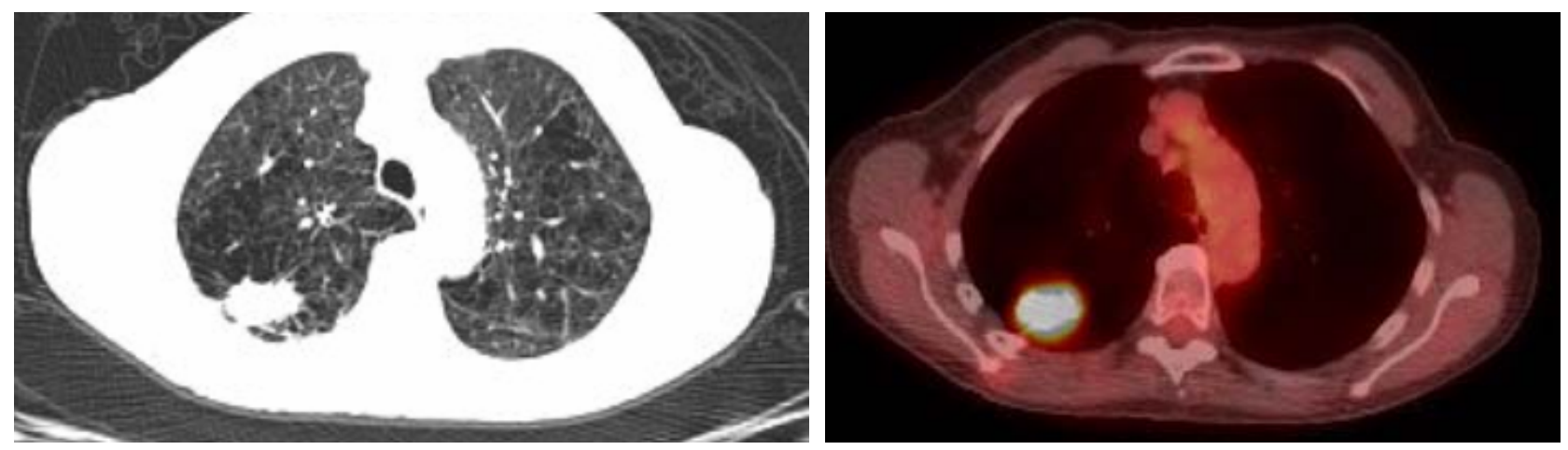

Image A. CT scan (left) and PET (right) showed a right upper lobe speculated mass $(2.8 \mathrm{~cm})$.
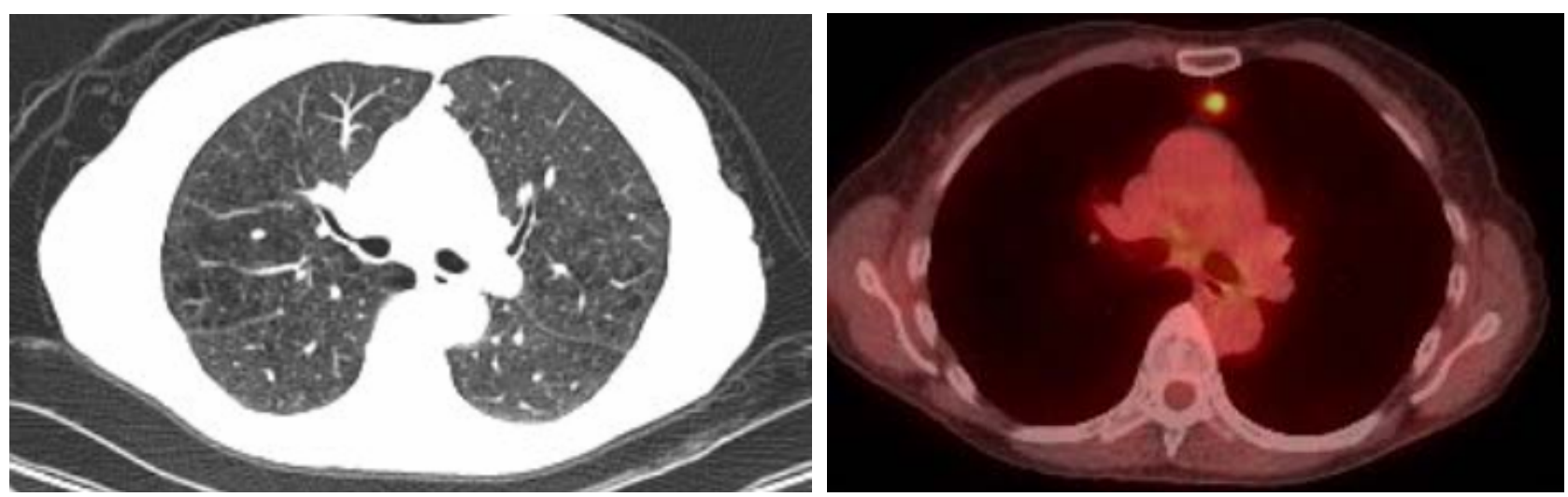

Image B. CT scan (left) PET (right) showed $16 \mathrm{~mm}$ mass on the medial left upper lobe.

\section{Details of Alzandi Technique for Bilateral Lung Masses}

This technique gives access to both the hemi thorax, and it can be done through the right or left chest. It is mainly for upper lobe lesions near the mediastinum, after either upper or lower lobectomy. The technique can be applied only to select types of patients. Previous anterior mediastinal or contralateral lung surgeries, right heart enlargement, and thymus tumor are to be considered relative contraindications for this approach in my opinion.

\section{Position}

After right upper lobectomy, patient is to continue in the lateral decubitus position, with significant posterior tilt to allow gravity to make the lung fall posterior, and out of the way.

\section{Step 1:}

\section{Incisions:}

The first incision: is placed at the $7^{\text {th }}$ or $8^{\text {th }}$ intercostal space, anterior to mid-axillary line, $10 \mathrm{~mm}$ port (for camera).

Second incision: $4-5 \mathrm{~cm}$ in length and is placed at approximately a 2 finger breadth from the latissmus dorsi border, one intercostals space above the superior pulmo- nary vein (utility port).

Third incision: is placed just below the inframammary crease as far anteriorly and inferiorly as possible (approximately $6^{\text {th }}$ intercostals space midaxillary line).

Fourth incision: $1 \mathrm{~cm}$ long incision placed at the auscultatory triangle, three finger breadth below the scapula. Sometimes we switch the camera through this port and we use the camera port for retracting the lung posterior.

\section{Step 2:}

Dissection: aim the scope superiorly, and point the lens toward the mediastinum. A grasper through the incision (No.3) to lift the pleura superior to superior vena cava, the phrenic nerve, and inferior to the right mammary pedicle. Then hook open the mediastinal pleura parallel to the right internal mammary pedicle (Figure 1). Bluntly create the plane between the peristernal tissue (including the mammary pedicle) and the left sided mediastinal pleura (avascular plane) (Figure 2). When medistinal pleura is clearly identified over the left lung, open the pleura widely with L-hook (Figure 3). The medial border of left lung and anterior surface will be very clear (Figure 4).

The lesion can be grasped and divided with endoGIA. If there are any issues regarding visualization of the lung lesion or the left phrenic nerve, add $5^{\text {th }}$ port in the left $7^{\text {th }}$ 


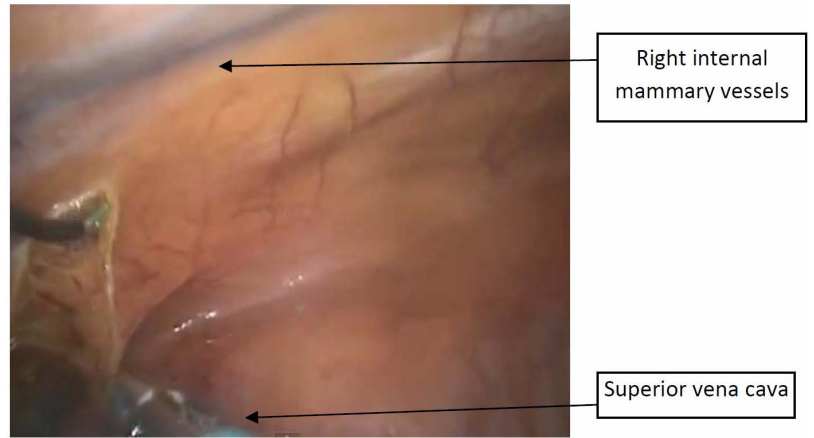

Figure 1. Showed the hook open the mediastinal pleura parallel to the right internal mammary pedicle.

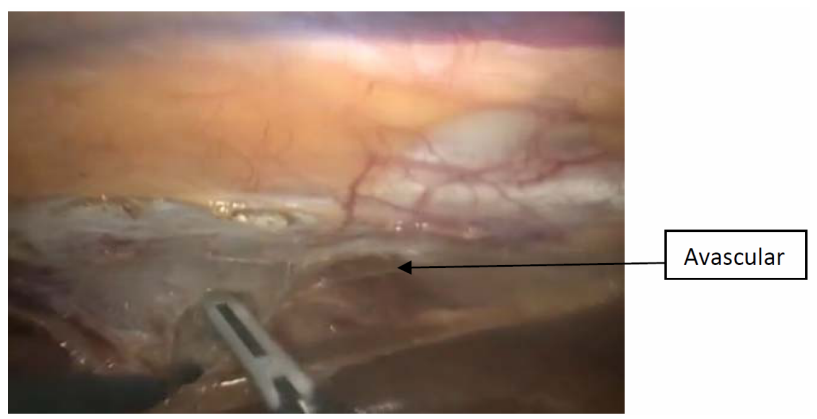

Figure 2. demonstrated the plane between the peristernal tissue (including the mammary pedicle) and the left sided mediastinal pleura (avascular plane).

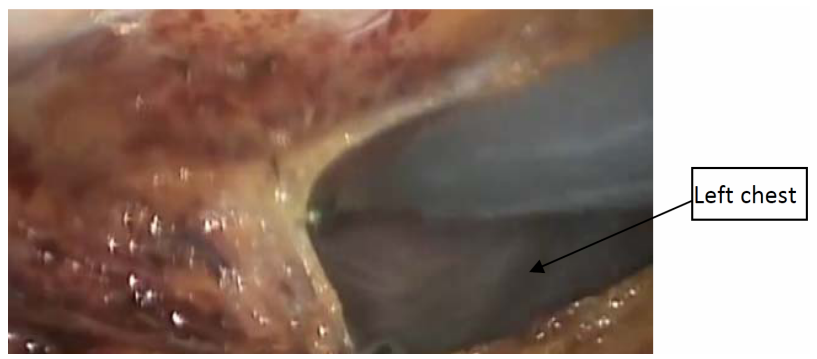

Figure 3. showed medistinal pleura over the left lung widely open with L-hook.

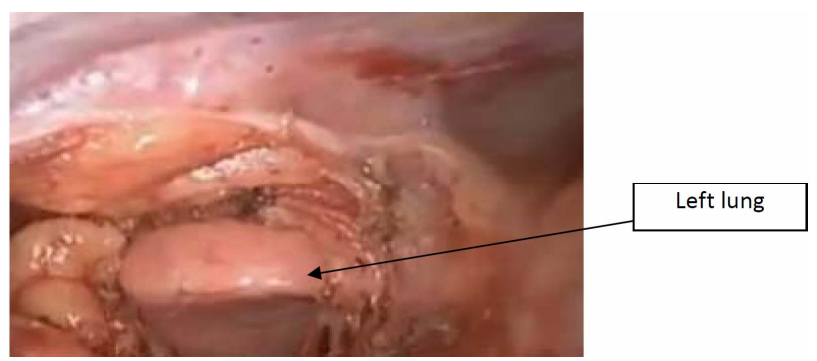

Figure 4. demonstrated the medial border of left lung and anterior surface.

intercostals space in anterior clavicular line. Put the camera in this port and ask the anesthesiologists to intermittently hold ventilation to visualize structures. Also, en- doscopic stapler can be introduced through this port without intraoperative posture change. At the end of the procedure, two $32 \mathrm{~F}$ chest tubes are inserted through the right-sided incisions to drain the right pleural cavity and to the left chest to drain the left plural space.

\section{Discussion}

The detection rate of synchronous multiple lung tumors has increased due to introduction of helical CT in the screening of lung cancers [1,2]. Prevalence of multiple primary lung cancers varies in the literature from $0.8 \%$ to $10.0 \%$ for both synchronous and metachronous cancers [3]. For synchronous multiple cancers alone, prevalence varies from $0.3 \%$ to $4.6 \%$.

Patients with a single contralateral lung lesion should not be treated as disseminated disease (stage IV). After extensive searching for metastatic spread, bilateral surgical resection should be considered in fit patients [4]. In the database of the IASLC, the median survival of 362 patients with bilateral synchronous lung cancer was 10 months, with the 1-year survival rate at $45 \%$ and the 5year survival rate at only 3\% [5]. This was significantly better compared to survival of patients with metastases outside the lung (median survival of 6 months). Based on these figures, the lung cancer staging committee proposes that patients with bilateral lung nodules should be staged as M1a, and patients with distant metastases should be designated M1b.

Wedge or segmental resection was an acceptable alternative for patients with bilateral lung cancer who were unable to tolerate bilateral anatomical resection. The use of a limited resection procedure did not seem to have negative effects on survival [4].

Bilateral staged thoracotomy, trans-sternal bilateral thoracotomy for bilateral lung lesions, and staged bilateral VATS are all possible options for treating patients with bilateral lung cancer. From here we did resection of bilateral lung lesion through the same incisions from one side and using the minimally invasive method. We believe extensive resection can be done through this approach, and the surgeon should try it in selected patients. The advantages of this approach may include less morbity (e.g. pain), decrease operative time, no need for repositioning and more cosmetic outcome. The approach is easily accessible by well trained thoracic surgeon. Limited exposure to contra lateral lung and potential injury to mediastinal structures can be minimized by adding another contralaterl port as described above.

Lobectomy started on the side where there was doubt as to the extent of resection needed, and/or possibility of complete resectability. This is usually the side of the largest lesion. If a complete resection can be achieved, 
the contralateral wedge resection is performed.

\section{Conclusion}

Although this new VATS access for bilateral lung cancer may not be widely advertised or adopted due to several controversies so far, it is technically feasible as one of the minimally invasive surgical options.

\section{REFERENCES}

[1] H. Beyreuther, "Multiplicitat von Carcinomen bei einem Fall von sog: 'Schneeberger', Lungenkrebs mit Tuberkulose," Virchows Archiv, Vol. 250, No. 1-2, 1924, pp. 230243. doi:10.1007/BF01891568

[2] K. Iino, M. Oda, Y. Tsunezuka, et al., "Treatment for Bilateral Multiple Lung Cancers,” Kyobu Geka, Vol. 55, No. 6, 2002, pp. 443-448.
[3] P. C. van Bodegom, S. S. Wagenaar, B. Corrin, J. P. Baak, J. Berkel and R. G. Vanderschueren, "Second Primary Lung Cancer: Importance of Long Term Follow up,” Thorax, Vol. 44, No. 10, 1989, pp. 788-793. doi:10.1136/thx.44.10.788

[4] P. De Leyn, J. Moons, J. Vansteenkiste, E. Verbeken, D. Van Raemdonck, P. Nafteux, H. Decaluwe and T. Leru, "Survial after Resection of Synchronous Bilateral Lung Cancer," Journal of Clinical Oncology, Vol. 26, No. 7, 2008, pp. 1128-1134.

[5] P. Postmus, E. Brambilla, K. Chansky, J. Crowley, P. Goldstraw, E. Patz and H. Yokomise, "The IASLC Lung Cancer Staging Project: Proposals for Revision of the M Descriptors in the Forthcoming (Seventh) Edition of the TNM Classification of Lung Cancer," Journal of Thoracic Oncology, Vol. 2, No. 8, 2007, pp. 686-693. doi:10.1097/JTO.0b013e31811f4703 